\title{
PREVALÊNCIA DO DIABETES MELLITUS GESTACIONAL EM UM AMBULATÓRIO DE ALTO RISCO DO OESTE DO PARANÁ
}

\section{PREVALENCE OF GESTATIONAL DIABETES MELLITUS IN A HIGH RISK OUTPATIENT CLINIC OF WEST PARANÁ}

\author{
Taís Cristina Rossett ${ }^{1 *}$, Thayline Wittmann ${ }^{1}$, Kauana Rotta ${ }^{1}$, Rafael Arneiro \\ Gonçalves ${ }^{1}$, Marise Vilas Boas Pescador ${ }^{2}$
}

\begin{abstract}
${ }^{1}$ Acadêmico do oitavo período de Medicina do Centro Universitário Fundação Assis Gurgacz. ${ }^{2}$ Médica Endocrinologista, Mestre em Saúde da Criança e do Adolescente, Professora orientadora e Docente do Centro Universitário Fundação Assis Gurgacz.

* Autor correspondente: taiscristina@hotmail.com, https://orcid.org/0000-0003-1341-8230
\end{abstract}

DOI: $10.35984 /$ fjh.v2i2.193

\section{RESUMO}

O Diabetes Mellitus Gestacional (DMG) é definido pelo aumento nos níveis da glicemia, detectado pela primeira vez durante a gestação, que cursa com complicações maternas e fetais. O presente estudo, objetivou avaliar a prevalência do DMG em um ambulatório especializado e os possíveis fatores de risco associados. Trata-se de um estudo quantitativo, de caráter descritivo-observacional realizado de maneira retrospectiva no Ambulatório de Alto Risco da Ginecologia e Obstetrícia do Centro de Atendimento Especializado (CAE), na cidade de Cascavel-PR. A população analisada foi constituída por todas as gestantes com idade superior a 18 anos, portadoras de DMG atendidas e diagnosticadas entre Janeiro e Dezembro de 2018. Foram selecionados 148 prontuários e destes 14 foram excluídos devido a equívoco de diagnóstico ou DM franco. A prevalência de DMG foi de $18,35 \%$, sendo que $37,71 \%$ das gestantes se encontravam na faixa etária de 25 a 30 anos, 59,7\% autodenominaram-se de raça branca; $50,76 \%$ eram obesas; $76,11 \%$ eram multíparas; $44,77 \%$ foram diagnosticadas no terceiro trimestre da gestação, 4,47\% possuíam diagnóstico de DMG em gestações anteriores, 97,01\% apresentavam pelo menos 1 fator de risco para DMG e 83,58\% apresentavam 2 ou mais fatores de risco. Sendo assim, é importante atentar-se às pacientes com qualquer fator de risco, a fim de diagnosticar e tratar precocemente a doença, evitando complicações. Palavras-chave: Diabetes Mellitus Gestacional. Prevalência. Epidemiologia. Fatores de Risco.

\begin{abstract}
An increase in blood glucose levels, detected for the first time during pregancy and that goes with maternal and fetal complications, defines Gestational Diabetes Mellitus (GDM). The present study, aimed to evaluate GDM prevalence and potential risk factors in a high risk outpatient clinic. This is a quantitative, descriptive-observational and retrospective study developed at the Ginecology and Obstetrics High Risk Outpatient Clinic of the Centro de Atendimento Especializado (CAE), in the city of Cascavel-PR. Pregnant women, older than 18 years, diagnosed with GDM between January of 2018 and December of 2018 were in wich the study was based. Were selected 148 medical records, but 14 were excluded because of wrong diagnosis or due to frank diabetes. The prevalence was about $18,35 \%$, and $37,71 \%$ of the patients were between ages 25 and 30 years old, 59,7\% called themselves white race, $50,76 \%$ were obese or overweight, $76,11 \%$ were multiparous women, $44,77 \%$ were diagnosed during the third trimester of pregnancy, 4,47\% has previous diagnosis of GDM, 97,01\% had at least one risk factor for GDM and 83,58\% of them had 2 or more risk factors. Therefore, it is important to pay attention to patients with any risk factor, in order to diagnose and treat the disease early and to avoid more complications.
\end{abstract}

Keywords: Gestational Diabetes Mellitus. Prevalence. Epidemiology. Risk factors. 


\section{INTRODUÇÃO}

A Sociedade Brasileira de Diabetes (SBD) define o Diabetes Mellitus Gestacional (DMG) como uma doença caracterizada por intolerância a carboidratos, identificada pela primeira vez durante a gestação e que pode manter-se após o término da mesma (SBD, 2014). É um dos principais distúrbios metabólicos da gestação e que tem prevalência estimada entre 3 e $25 \%$, pois varia dependendo da população estudada e do critério diagnóstico utilizado (SBD, 2017). Estima-se que no Sistema Único de Saúde (SUS), a prevalência do DMG esteja próxima de 18\% (OPAS, 2017). De acordo com os últimos estudos realizados mundialmente constatou-se que a cada 100 mulheres, 16 delas desenvolveram diabetes durante a gestação (OPAS, 2017).

A gestação é um processo que envolve uma série de mudanças fisiológicas no organismo feminino a fim de atender às demandas do feto, uma importante adaptação metabólica que ocorre nesse período e que interfere no desenvolvimento do DMG é a resposta à insulina (PLOWS et al, 2018). Comparando-se uma gestante normal com uma gestante portadora de DMG, nota-se que as últimas apresentam uma resposta insulínica menor (KAAJA; RONNEMAA, 2008).

Hodson, Robson e Taylor (2010), afirmam que a insulina é produzida nas células $\beta$-pancreáticas quando há um aumento nos níveis de glicose sanguíneos. Plows et al (2018), acrescenta que no início da gestação, há uma produção ainda maior de insulina, a ser armazenada, como forma de preparação do organismo para as demandas do feto que está em desenvolvimento. Conforme a gestação avança, entre 18 e 28 semanas, hormônios placentários como o lactogênio e a progesterona, associados a ação do cortisol e da prolactina acabam neutralizando os efeitos da insulina e levando ao estado de resistência (HODSON; ROBSON; TAYLOR, 2010). Ademais, a gestação é naturalmente um estado pró-inflamatório que envolve a produção de citocinas as quais também contribuem para a resistência insulínica (HODSON; ROBSON; TAYLOR, 2010).

Oliveira et al. (2014), salienta que a obesidade também é interpretada como um estado crônico de inflamação, pois no tecido adiposo, por estímulo dos macrófagos, também haverá a produção de citocinas tais como o fator de necrose tumoral e interleucina 1, as quais atuam como inibidores da insulina. No que tange à obesidade, Hodson, Robson e Taylor (2010) sugerem que outro fator que parece estar associado ao DMG é a baixa concentração de adiponectina, uma proteína sintetizada pelo tecido adiposo responsável por estimular o armazenamento de glicose dentro do músculo esquelético e inibir sua produção. Estudos têm mostrado que com o progresso de uma gestação normal os níveis de adiponectina tendem a reduzir e em casos de DMG esses níveis estão ainda mais reduzidos, resultando em um aumento da glicose sanguínea (HODSON; ROBSON; TAYLOR, 2010).

Segundo Plows et al. (2018), os principais fatores de risco para o DMG são sobrepeso, ganho excessivo de peso durante a gestação, etnia, fatores genéticos, idade materna avançada, antecedentes pessoais ou familiares de DMG e outras doenças que cursam com resistência insulínica como a síndrome do ovário policístico.

O diagnóstico do DMG geralmente é feito entre o segundo e terceiro trimestres, quando a resistência insulínica fica mais evidente (OPPERMAN; GENRO; REICHELT, 2018). Uma grande parcela das mulheres desenvolve a intolerância à glicose apenas durante a gestação, mas algumas pacientes podem ter diabetes prévio não diagnosticado (OPPERMAN; GENRO; REICHELT, 2018).

Os guidelines recentes recomendam 0 rastreamento em mulheres que apresentam, principalmente, IMC > 30, gestação prévia cujo concepto nasceu com FAG Journal of Health - ISSN 2674-550X, 2020, v.2, n.2, p. 196

DOI: 10.35984/fjh.v2i2.193 
$4500 \mathrm{~g}$ ou mais, DMG em gestações anteriores ou história familiar de diabetes (HODSON; ROBSON; TAYLOR, 2010).

Todo médico deve solicitar já na primeira consulta do pré-natal uma glicemia de jejum. Quando o resultado do exame for $\geq 92 \mathrm{mg} / \mathrm{dL}$ e $<126 \mathrm{mg} / \mathrm{dL}$, em duas dosagens, é possível estabelecer o diagnóstico de DMG, e se a glicemia for $<92$ $\mathrm{mg} / \mathrm{dL}$, deve-se reavaliar a gestante no segundo trimestre e realizar o teste oral de tolerância à glicose (TOTG) no terceiro trimestre entre a $24^{\mathrm{a}}$ e $28^{\mathrm{a}}$ semana. Esse apresenta como pontos de corte valores de glicose em jejum $\geq 92 \mathrm{mg} / \mathrm{dL}$ e $\geq 180$ $\mathrm{mg} / \mathrm{dL}$ e $153 \mathrm{mg} / \mathrm{dL}$ com 1 e 2 horas após o uso de 75 gramas de glicose, respectivamente. Apenas um valor anormal já permite o diagnóstico de DMG. Entretanto, nos casos em que a dosagem da glicemia em jejum for $\geq 126 \mathrm{mg} / \mathrm{dL}$ ou após 2 horas do teste de sobrecarga o valor for $>200 \mathrm{mg} / \mathrm{dL}$ o diagnóstico será de diabetes mellitus franco na gravidez (SBD, 2017).

A primeira linha de tratamento do diabetes gestacional é a terapia nutricional, que visa atenuar os efeitos do excesso de peso sobre a doença, bem como reduzir a incidência de complicações como a macrossomia fetal e outras complicações perinatais (WEINERT et al., 2011). O consumo calórico médio diário indicado para gestantes de baixo peso é de $35 \mathrm{kcal} / \mathrm{kg} / \mathrm{dia}$, se o peso for adequado é de $30 \mathrm{kcal} / \mathrm{kg}$, ao passo que gestantes obesas devem ter uma ingesta calórica de até $25 \mathrm{kcal} / \mathrm{kg} / \mathrm{dia}$ (MARUICHI; AMADEI; ABEL, 2012). Deve-se optar por alimentos com baixo índice glicêmico e estimular um consumo balanceado entre carboidratos (40 - 55\%), proteínas (15 - 20\%) e gorduras (30-40\%) (SBD, 2017).

Em associação às alterações dietéticas, deve-se estimular a prática de exercícios físicos de acordo com orientações obstétricas (SBD, 2017). A atividade física além de reduzir gordura aumenta a afinidade entre a insulina e o seu receptor atuando de forma favorável na resistência insulínica (MARUICHI; AMADEI; ABEL, 2012). A glicemia capilar pré e pós-prandial deve ser monitorada diariamente, de quatro a sete vezes, por no mínimo 2 semanas durante o tratamento nãofarmacológico (SBD, 2017). O tratamento farmacológico será instituído se, mesmo com medidas dietéticas e prática de atividades físicas, os níveis de glicose permanecerem superiores as metas estabelecidas $(<95 \mathrm{mg} / \mathrm{dL}$ em jejum e préprandial ou $<120 \mathrm{mg} / \mathrm{dL} 2$ horas pós-prandial) (CUNNINGHAMet al., 2018).

O tratamento de DMG padrão é a insulina, por ser eficaz e segura, mas recentemente os hipoglicemiantes orais têm sido utilizados (OPPERMAN; GENRO; REICHELT, 2018). Em geral os tipos de insulina utilizados são as de ação intermediária e rápida, podendo variar conforme a necessidade (SBD, 2017). Se ainda houver hiperglicemia no jejum, utiliza-se a insulina NPH em dose sugerida de 0,2 Ul/kg e se predominar a hiperglicemia pós-prandial, adota-se a insulina de ação rápida ou ultrarrápida, em dose de $1,5 \mathrm{UI}$ a cada $10 \mathrm{~g}$ de carboidratos ingeridos no café da manhã e no almoço e no jantar $1 \mathrm{UI}$ para cada $10 \mathrm{~g}$ de carboidrato ingerida (OPPERMAN; GENRO; REICHELT, 2018).

Apesar de a insulina ser a primeira linha de tratamento, muitos estudos têm evidenciado a segurança e a eficácia dos hipoglicemiantes orais (CUNNINGHAMet al., 2018). A metformina, apesar de ter passagem placentária, se utilizada no primeiro trimestre de gestação, não exerce influência sobre malformações congênitas (OPPERMAN; GENRO; REICHELT, 2018). Já a glibenclamida, apesar de ter pequena passagem placentária, pode estar associada a riscos maiores de macrossomia e hipoglicemia neonatal (OPPERMAN; GENRO; REICHELT, 2018).

As complicações estão presentes desde a concepção até o período pósgestacional e no primeiro trimestre de gestação o DMG pode provocar disfunções no FAG Journal of Health - ISSN 2674-550X, 2020, v.2, n.2, p. 197

DOI: $10.35984 /$ fjh.v2i2.193 
desenvolvimento dos órgãos, malformações e, inclusive, abortamento (AMARAL et al., 2012). Já ao longo da gestação, a complicação mais atribuída ao DMG é a macrossomia ou crescimento fetal excessivo (CUNNINGHAM et al., 2018).

Ao longo da gestação a glicose passa da mãe para o feto, fazendo com que ele também desenvolva hiperglicemia e responda a esse estado com a hiperinsulinemia fetal, ou seja, o excesso de produção de insulina faz com que a glicose do feto ao nascer seja rapidamente metabolizada provocando um quadro de hipoglicemia neonatal (BOLOGNANI; SOUZA; CALDERON, 2011). Além das adversidades fetais, as mães também apresentam risco elevado de pré-eclâmpsia, hipertensão gestacional e de diabetes mellitus tipo 2 (DM2) após a gestação, sendo que aproximadamente $50 \%$ das gestantes com DMG serão diagnosticadas com diabetes tipo 2 (OPPERMAN; GENRO; REICHELT, 2018).

Os desfechos adversos não se restringem apenas à gestação. Estima-se que o DMG é um fator predisponente de obesidade e de falhas no desenvolvimento psicomotor do concepto, futuramente (AMARAL et al., 2012). O principal fator associado ao surgimento da obesidade na infância e também da síndrome metabólica é a macrossomia (BOLOGNANI; SOUZA; CALDERON, 2011). Tendo em vista a incidência de DMG na população e as complicações decorrentes dessa endocrinopatia, o presente estudo tem como finalidade identificar a sua prevalência e o perfil das gestantes portadoras dessa patologia em um ambulatório de Alto Risco. Os aspectos analisados foram a idade, a raça, o IMC gestacional, a paridade, 0 trimestre em que a doença foi diagnosticada e história prévia de DMG

\section{METODOLOGIA}

Este trabalho, por se tratar de pesquisa com seres humanos está em cumprimento com a Resolução 466/12 do Conselho Nacional de Saúde. A pesquisa foi aprovada pelo Comitê de Ética em Pesquisa do Centro Universitário Fundação Assis Gurgacz com o Certificado de Apresentação para Apreciação Ética (CAAE) de número 14384819.5219.

Tratou-se de um estudo quantitativo, de caráter descritivo-observacional realizado de maneira retrospectiva, com pacientes atendidas no Centro de Atendimento Especializado (CAE) de Cascavel-PR. Foram incluídas no estudo, todas as gestantes atendidas e diagnosticadas com DMG no período de Janeiro de 2018 a Dezembro de 2018 e que possuíam resultados de pelo menos um exame de glicose em jejum ou de um teste de tolerância oral à glicose. Foram excluídas do estudo, todas as gestantes menores de 18 anos de idade, além de gestantes com diagnóstico prévio de Diabetes Mellitus. $O$ instrumento dessa coleta de dados se baseou na análise de prontuários das gestantes diagnosticadas com DMG, os aspectos analisados foram a idade, a raça, o peso, a altura, a paridade, o trimestre em que a doença foi diagnosticada e história prévia de DMG. Para obtenção dos resultados foram utilizados todos os prontuários que se encontravam no Ambulatório de Alto Risco da Ginecologia e Obstetrícia do CAE, do período de Janeiro a Dezembro de 2018 e os dados encontrados foram comparados com os existentes na literatura.

Para o diagnóstico de DMG foram considerados os critérios diagnósticos propostos pela Sociedade Brasileira de Diabetes referentes ao ano de 2017. Para avaliar a prevalência foram consideradas todas as pacientes em acompanhamento no ambulatório de alto risco e selecionadas as que possuíam diagnóstico de DMG; para análise da idade, as pacientes foram divididas por faixa etária com intervalo de 5 anos e os resultados foram expressos por meio de média \pm desvio padrão.

FAG Journal of Health - ISSN 2674-550X, 2020, v.2, n.2, p. 198

DOI: $10.35984 /$ fjh.v2i2.193 
A classificação do estado nutricional foi realizada através do índice de massa corporal (IMC) adequado para a idade gestacional conforme preconizado pelo Ministério da Saúde. Foram considerados como fatores de risco para DMG: idade igual ou superior a 25 anos, sobrepeso ou obesidade, multiparidade e presença de DMG em gestação prévia. Os dados coletados foram tabulados em planilha do Excel e analisados por meio de estatística descritiva e os resultados foram apresentados sob forma de tabelas e gráficos.

\section{RESULTADOS E DISCUSSÃO}

Em um total de 730 prontuários de gestantes em acompanhamento no ambulatório de alto risco do CAE, foram inicialmente incluídos no estudo 148. Desses, 8 foram excluídos por apresentarem critérios para DM franco e outros 6 por equívoco de diagnóstico. Totalizando assim, 134 gestantes que compuseram o estudo e cujos dados analisados estão relacionados na tabela 1.

Tabela 1. Dados analisados nos prontuários de portadoras de DMG

\begin{tabular}{lcc}
\hline & $\mathbf{1 0 0} \% \mathbf{( N ~ = ~ 1 3 4 )}$ & \\
\hline CARACTERíSTICA & $\mathbf{N}($ total 134$)$ & $\%(100)$ \\
Faixa Etária & 27 & $20,14 \%$ \\
$19-24$ & 50 & $37,31 \%$ \\
$25-30$ & 37 & $27,61 \%$ \\
$30-35$ & 16 & $11,94 \%$ \\
$36-40$ & 4 & $2,98 \%$ \\
$41-45$ & & \\
Raça & 80 & $59,70 \%$ \\
Branca & 38 & $28,35 \%$ \\
Parda & 8 & $5,97 \%$ \\
Negra & 2 & $1,94 \%$ \\
Amarela & 6 & $4,47 \%$ \\
Ignorado & 26 & \\
Paridade & 102 & $19,40 \%$ \\
Primípara & 6 & $76,11 \%$ \\
Multípara & & $4,47 \%$ \\
Ignorado & 53 & $39,55 \%$ \\
Diagnóstico de DMG & 21 & $15,67 \%$ \\
Primeiro trimestre & 60 & $44,77 \%$ \\
Segundo trimestre & & \\
Terceiro trimestre & 6 & $4,47 \%$ \\
DMG prévio & 128 & $95,52 \%$ \\
Sim & & \\
Não & & \\
\hline
\end{tabular}

Dos 730 prontuários analisados, 134 gestantes possuíam DMG representando uma prevalência de $18,35 \%$. Os resultados encontrados nesse estudo demonstram um percentual superior ao encontrado em um estudo semelhante realizado por Dias, et al (2014) no Tocantins, que foi de $6 \%$. Tendo em vista, que foram analisadas populações em diferentes regiões do Brasil os resultados vão ao encontro do proposto na literatura em que a prevalência pode variar entre 1 e $37,7 \%$ de acordo com a população a ser estudada e também conforme os critérios diagnósticos utilizados (OPAS, 2017). Os diferentes critérios diagnósticos utilizados atualmente estão presentes na tabela 2. 
Tabela 2. Critérios Diagnósticos de DMG baseados nos resultados do TOTG com ingestão de $75 \mathrm{~g}$ de glicose.

\begin{tabular}{lccc} 
& OMS (2013) & NIH (2012)* & $\begin{array}{c}\text { IADPSG (2010)**; ADA e SBD (2011); SBD, }^{\star *} \\
\text { OPAS, FEBRASGO e MS (2017) }\end{array}$ \\
\hline Jejum & 92 a $125 \mathrm{mg} / \mathrm{dL}$ & $95 \mathrm{mg} / \mathrm{dL}$ & $92 \mathrm{mg} / \mathrm{dL}$ \\
1 hora & $180 \mathrm{mg} / \mathrm{dL}$ & $180 \mathrm{mg} / \mathrm{dL}$ & $180 \mathrm{mg} / \mathrm{dL}$ \\
2 horas & 153 a $199 \mathrm{mg} / \mathrm{dl}$ & $155 \mathrm{mg} / \mathrm{dL}$ & $153 \mathrm{mg} / \mathrm{dL}$ \\
\hline
\end{tabular}

OMS: Organização Mundial da Saúde; NIH: National Institutes of Health; IADPSG: International Association of the Diabetes and Pregnancy Groups; ADA: American Diabetes Association; SBD: Sociedade Brasileira de Diabetes; OPAS: Organização Pan Americana da Saúde; FEBRASGO: Federação Brasileira das Associações de Ginecologia e Obstetrícia; MS: Ministério da Saúde. * Dois valores alterados confirmam o diagnóstico. ${ }^{* *}$ Um valor alterado confirma o diagnóstico.

Fonte: Diretrizes SBD (2017)

Dentre as pacientes analisadas, 20,14\% (27) estavam na faixa etária entre 19 e 24 anos, $37,31 \%(50)$ entre 25 e 30 anos, $27,61 \%$ (37) entre 31 e 35 anos, $11,94 \%$ (16) entre 36 e 40 anos e 2,98\% (4) entre 41 e 45 anos. Na pesquisa realizada por Oliveira e Graciliano (2015), 50\% das gestantes encontravam-se na faixa etária entre 20-34 anos. Da mesma forma, os dados encontrados nesse estudo demonstram que $76,8 \%$ (103) das participantes encontravam-se nessa mesma faixa etária. Além disso, a idade média das pacientes foi de 29,7 $\pm 5,6$, sendo a idade mínima 19 anos e a máxima 42 anos. Nogueira, et al (2011), encontrou em seu estudo uma idade média das gestantes que evoluíram com DMG de 34,4 anos. Tais dados estão de acordo com os expostos pela literatura, considerando que o risco de desenvolver a doença aumenta com a idade e principalmente em gestantes acima de 25 anos (DIAS et al, 2014 e OPAS, 2017).

Também foi analisada a etnia das pacientes, sendo a branca a mais prevalente $59,7 \%(80)$, seguida pela parda $28,36 \%$ (38), negra $5,97 \%$ (8) e amarela com $1,49 \%$ (2). A raça de 6 pacientes foi ignorada pela falta dos dados nos prontuários. A maior parte da população estudada por Nogueira, et al (2011), tinha a cor da pele classificada como faioderma (62\%). Entretanto, o presente estudo se mostrou mais concordante com a pesquisa feita por Oliveira e Graciliano (2015) cujo grupo estudado era, em sua maioria, de cor branca/parda (92,8\%). Entretanto, Bolognani, et al (2011) afirma que, apesar de existirem dados na literatura que incluem a etnia como fator de risco, a população brasileira é extremamente miscigenada o que dificulta estabelecer uma relação entre a etnia e o DMG.

Em relação ao Índice de Massa Corporal (IMC), as gestantes foram classificadas com base em medidas obtidas na primeira consulta, utilizando-se os critérios de IMC propostos pelo Ministério da Saúde (MS) durante a gestação (Gráfico 1). Observouse que $23,14 \%$ (31) estavam com sobrepeso, enquanto $50,76 \%$ (68) das gestantes eram obesas. O IMC de 25 gestantes foi ignorado por falta de dados no prontuário. $O$ sobrepeso e a obesidade são considerados fatores de risco para o DMG, o que pôde ser visto no estudo de Oliveira e Graciliano (2015), cujos resultados concluíram que $61,5 \%$ das gestantes apresentavam sobrepeso ou obesidade baseando-se no IMC gestacional e estando de acordo com a presente pesquisa que encontrou 73,9\% (99) das gestantes apresentando sobrepeso ou obesidade.

No que tange à paridade, demonstrou-se que a maioria das gestantes eram multíparas $76,11 \%$ (102) e dentre elas 46,07\% (47) já apresentavam 3 ou mais gestações, enquanto 19,4\% (26) eram primíparas. Segundo Massucati, Pereira e Maioli (2012), não existem evidências de que a multiparidade seja um fator de risco para o DMG, porém seu estudo observou uma maior prevalência da doença em gestantes que apresentavam 3 gestações ou mais. Da mesma forma, Oliveira e 
Graciliano (2015), concluíram que $71,4 \%$ das gestantes eram multíparas. Mesmo sem ter uma correlação estabelecida, os dados obtidos nesse estudo estão em concordância com os estudos citados anteriormente.

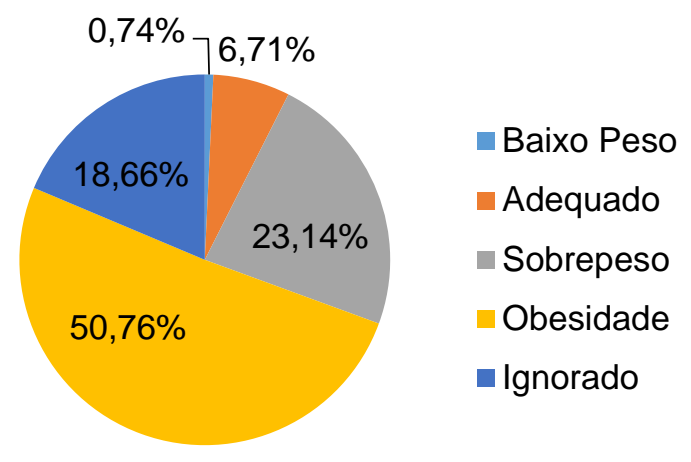

Figura 1 - Índice de Massa Corporal Gestacional das pacientes atendidas no ambulatório de alto risco do CAE

Ao analisar a idade gestacional em que foi realizado o diagnóstico de DMG, observou-se que $44,77 \%(60)$ das gestantes foram diagnosticadas durante o terceiro trimestre de gestação, $39,55 \%$ (53) no primeiro trimestre e 15,67\% (21) no segundo trimestre. Esse resultado corrobora com a hipótese de Hodson, Robson e Taylor (2010) que afirmam que entre o segundo trimestre e início do terceiro há maior ação dos hormônios placentários levando à resistência insulínica. Da mesma forma, na revisão feita por Massucati, Pereira e Maioli (2012), a maioria dos diagnósticos foram feitos no terceiro trimestre de gestação, estando de acordo com o resultado encontrado nessa pesquisa

A presença de DMG em gestações anteriores também foi avaliada, encontrando uma prevalência de 4,47\% (6). A história prévia ou familiar de DMG é citada na literatura como um fator de risco (SIMON, MARQUES e FARHAT, 2013 e PLOWS et al 2018). Contudo, Dias, et al (2014) analisando sua população, observou que apenas $4 \%$ apresentavam história prévia de DMG. Da mesma forma, o estudo atual mostrou que a ocorrência de DMG em gestação prévia foi baixa.

Por fim, realizou-se uma análise acerca da relação entre fatores de risco e a prevalência da doença, sendo observado que $97,01 \%$ (130) gestantes apresentavam pelo menos 1 fator de risco e $83,58 \%$ (112) delas apresentavam 2 ou mais fatores de risco (tabela 3). Os resultados encontrados vão ao encontro de um estudo sobre o perfil e evolução de um grupo de pacientes de um hospital do Estado de Minas Gerais, onde em uma amostra de 66 pacientes $88 \%$ delas apresentavam pelo menos um fator de risco de DMG.

Tabela 3. Relação dos fatores de risco presentes na amostra

\begin{tabular}{lcc}
\hline & $\mathbf{1 0 0} \%(\mathbf{N}=\mathbf{1 3 4})$ & \\
\hline FATOR DE RISCO & $\mathrm{N}$ & $\%$ \\
Idade $\geq 25$ anos & 107 & $79,85 \%$ \\
Sobrepeso ou obesidade & 99 & $73,88 \%$ \\
Multiparidade & 102 & $76,11 \%$ \\
DMG prévio & 6 & $4,47 \%$ \\
\hline
\end{tabular}




\section{CONSIDERAÇÕES FINAIS}

A análise das informações obtidas nos prontuários das gestantes atendidas no ambulatório de alto risco do CAE de Cascavel-PR permitiu evidenciar uma incidência de DMG de $18 \%$ nesse grupo, sendo mais prevalente na faixa etária entre 25 e 30 anos. Também foi encontrado uma alta incidência de sobrepeso e/ou obesidade, assim como, a maioria das gestantes apresentavam um ou mais fatores de risco para o desenvolvimento de DMG. A partir desses dados, pôde-se observar que a prevalência da doença estudada está dentro do esperado, mas superior a estudos realizados em outras regiões do país e que há uma possível relação da prevalência com a idade superior a 25 anos, multiparidade, raça branca e presença de pelo menos um fator de risco para DMG, além do diagnóstico ser mais frequente no terceiro trimestre de gestação. Frente ao significante número de pacientes acometidas pelo DMG, concluiu-se que o rastreamento precoce e constante de gestantes com qualquer fator de risco é essencial no âmbito de realizar o diagnóstico e intervenção precoces e assim evitar futuras complicações materno fetais decorrentes dessa enfermidade.

\section{REFERÊNCIAS}

AMARAL, A.C.S et al. Complicações neonatais do diabetes mellitus gestacional DMG.Rev. Med. Minas Gerais v.22, (Supl 5), 2012. Disponível em http://rmmg.org/sumario/56. Acesso em: 13 dez. 2018.

BOLOGNANI, C.V; SOUZA, S. S; CALDERON, I. M. P .Diabetes mellitus gestacional - enfoque nos novos critérios diagnósticos. Com. Ciências Saúde. v.22, 2011. Disponível

em: $<$ http://bvsms.saude.gov.br/bvs/artigos/diabetes mellitus gestacional.pdf $>$. Acesso em: 19 jan. 2019.

Classificação do estado nutricional foi realizada através do índice de massa corporal (IMC) adequado para a idade gestacional conforme preconizado pelo Ministério da Saúde.Disponível em: <http://www.saude.gov.br/component/content/article/804imc/40512-imc-para-gestantes>. Acesso em: 8 set. 2019

\section{CUNNINGHAM, F. G, et al. Williams Obstetrics. 25.ed. 2018.}

DIAS, B.Fet al. Epidemiologia do Diabetes Mellitus Gestacional no Ambulatório de Alto Risco do SUS em Araguaína-TO em 2013. Revista Científica do ITPAC. v.7, n.3, 2014. Disponível em: $<$ https://assets.itpac.br/arquivos/Revista/73/artigo6.pdf> Acesso em: 12 jul. 2019.

HODSON, K; ROBSON, S; TAYLOR, R. Gestational diabetes: emergingconcepts in phatophysiology. Obstetric Medicine, v.3, 2010. Disponível em: https://www.ncbi.nlm.nih.gov/pmc/articles/PMC4989628/pdf/10.1258 om.2010.10002 5.pdf. Acesso em: 15 mar. 2019. DOI: 10.1258/om.2010.100025

KAAJA, R.; RONNEMAA, R. Gestational Diabetes: Pathogenesis and Consequences to Mother and Offspring. The Review of Diabetic Studies. v.5, n.4, 2008. Disponível em: https://www.ncbi.nlm.nih.gov/pmc/articles/PMC2664679/pdf/RevDiabeticStud-05194.pdf. Acesso em: 15 mar. 2019. DOI 10.1900/RDS.2008.5.194 
MARUICHI, M.D; AMADEI, G; ABEL, M.N.C. Diabetes mellitus gestacional. Arquivos Médicos dos Hospitais e da Faculdade de Ciências Médicas da Santa Casa de São Paulo. v.57, n.3, 2012. Disponível em: $<$ http://arquivosmedicos.fcmsantacasasp.edu.br/index.php/AMSCSP/article/view/296/ 308>. Acesso em: 16 mar. 2019.

NOGUEIRA, A.I et al. Diabetes Gestacional: perfil e evolução de um grupo de pacientes do Hospital das Clínicas da UFMG. RevMed Minas Gerais, v.21, n.1, 2011. Disponível em: <http://rmmg.org/artigo/detalhes/288>. Acesso em: 29 jun. 2019.

OLIVEIRA, A.C.M.; GRACILIANO, N.G. Síndrome Hipertensiva da Gravidez e Diabetes Mellitus Gestacional em uma maternidade pública de uma capital do nordeste brasileiro, 2013: prevalência e fatores associados. Epidemiol. Serv. Saúde, Brasília, v. 24, n.3, 2015. Disponível em: <http://www.scielo.br/pdf/ress/v24n3/22379622-ress-24-03-00441.pdf>. Acesso em: 10 jul. 2019. DOI: 10.5123/S167949742015000300010

OLIVEIRA, C. C. G, et al. Diabetes Gestacional Revisitada: Aspectos bioquímicos e fisiopatológicos. Revista Humano Ser. v.1, n.1, 2014. Disponível em: https://periodicos.unifacex.com.br/humanoser/article/view/571/148. Acesso em: 16 mar. 2019.

OPPERMAN, M. L. R; GENRO, V. K; REICHELT, A.J. Diabetes Melito e Gestação. In: COSTA, S. H. M. (Org). Rotinas em Obstetrícia. 7.ed. Porto Alegre: Artmed, 2018.

Organização Pan-Americana da Saúde. Ministério da Saúde. Federação Brasileira das Associações de Ginecologia e

Obstetrícia. Sociedade Brasileira de Diabetes. Rastreamento e diagnóstico de Diabetes mellitus gestacional no Brasil. - Brasília. 2017. Disponível em https://www.diabetes.org.br/profissionais/images/pdf/diabetes-gestacionalrelatorio.pdf Acesso em: 13 dez. 2018.

PLOWS, J. F, et al. The Pathophysiology of Gestational Diabetes Mellitus. InternationalJournalof Molecular Sciences. v.19, 2018. Disponível em; <https://www.ncbi.nlm.nih.gov/pmc/articles/PMC6274679/>. Acesso em: 15 mar. 2019. DOI: $10.3390 /$ ijms 19113342

SIMON, C.Y; MARQUES, M.C.C; FARHAT, H.L. Glicemia de jejum do primeiro trimestre e fatores de risco de gestantes com diagnóstico de diabetes melito gestacional. Rev. Brasileira de Ginecologia e Obstetrícia. v. 25, n.11, 2013. Disponível em: <http://www.scielo.br/pdf/rbgo/v35n11/v35n11a06.pdf>. Acesso em: 21 jun. 2019. https://doi.org/10.1590/S0100-72032013001100006

Sociedade Brasileira de Diabetes. Avaliação e tratamento do diabetes mellitus gestacional. In: Diretrizes Sociedade Brasileira de Diabetes 2017-2018. São Paulo: Clannad, 2017. Disponível em: $<$ https://www.diabetes.org.br/profissionais/images/2017/diretrizes/diretrizes-sbd2017-2018.pdf>. Acesso em: 19 jan. 2019. 
Sociedade Brasileira de Diabetes. DIRETRIZES SBD. Diabetes mellitus gestacional: diagnóstico, tratamento e acompanhamento pós-gestação. 20142015.

p. 192-197.

Disponível

em:

$<$ https://www.diabetes.org.br/profissionais/images/pdf/diabetes-gestacional/001-

Diretrizes-SBD-Diabetes-Gestacional-pg192.pdf>. Acesso em: 13 dez. 2018.

WEINERT, L.S et al. Diabetes gestacional: um algoritmo de tratamento multidisciplinar. ArqBrasEndocrinolMetabol. v.55, n.7, 2011.Disponível em: $<$ http://www.scielo.br/pdf/abem/v55n7/02.pdf>. Acesso em: 23 jun. 2019. https://doi.org/10.1590/S0004-27302011000700002 